Nutrition Science en évolution

La revue de l'Ordre professionnel des diététistes du Québec

\title{
Le secret professionnel : un droit réel, mais une obligation méconnue
}

\section{Janick Perreault}

Volume 16, numéro 2, automne 2018

URI : https://id.erudit.org/iderudit/1054345ar

DOI : https://doi.org/10.7202/1054345ar

Aller au sommaire du numéro

Éditeur(s)

Ordre professionnel des diététistes du Québec

ISSN

2561-620X (numérique)

Découvrir la revue

Citer cet article

Perreault, J. (2018). Le secret professionnel : un droit réel, mais une obligation méconnue. Nutrition Science en évolution, 16(2), 31-34.

https://doi.org/10.7202/1054345ar d'utilisation que vous pouvez consulter en ligne. 


\section{LESECRET PROFESSIONNEL: UN DROIT RÉEL, MAIS UNE OBLIGATION MÉCONNUE}

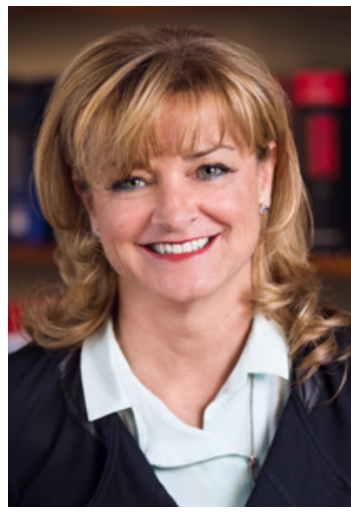

Le secret professionnel constitue une obligation de la plus grande importance pour l'ensemble des professionnels en raison des informations privilégiées obtenues pendant la relation avec leurs clients. Le sujet est suffisamment important pour que nous en traitions de nouveau ${ }^{[]}$afin d'en rappeler le fondement, la portée et les limites.

Maître Janick Perreault, Ad. E., Dt.P., LL.B., LL.M.*

\section{Le droit au secret professionnel}

Le droit québécois reconnaît depuis longtemps l'importance du secret professionnel. La protection des données de nature confidentielle est préservée par la Charte québécoise des droits et des libertés, de même que par le Code civil du Québec. À l'égard de la relation thérapeutique, la Loi sur l'accès aux documents des organismes publics et sur la protection des renseignements personnels ${ }^{[2]}$ ainsi que la Loi sur les services de santé et les services sociaux ${ }^{[3]}$ contiennent plusieurs dispositions visant à assurer la confidentialité des renseignements médicaux au sein des établissements de santé. Quant aux cabinets privés, ils sont assujettis aux dispositions prévues en la matière dans la Loi sur la protection des renseignements personnels dans le secteur privé ${ }^{[4]}$.

Le droit au respect du secret professionnel est un droit fondamental prévu par la Charte des droits et libertés de la personne ${ }^{[5]}$. Même devant la justice, toute personne tenue au secret professionnel ne peut pas divulguer de renseignements confidentiels qui lui ont été révélés en raison de sa profession à moins d'y être autorisée par celui qui leur a fait une confidence ou par une disposition expresse de la loi.

\section{Le secret professionnel est une obligation}

Le respect du secret professionnel est un droit fondamental qui appartient au client de toute personne membre d'un ordre professionnel et une obligation pour cette dernière. En effet, le Code des professions ${ }^{[6]}$ impose à tous les professionnels qu'il régit le devoir de respecter le secret de tout renseignement de nature confidentielle qui vient à sa connaissance dans l'exercice de sa profession ${ }^{[7]}$.

Le Code de déontologie des diététistes ${ }^{[8]}$ encadre spécifiquement le secret professionnel en réitérant que le diététiste/nutritionniste doit respecter le secret de tout renseignement de nature confidentielle obtenu dans l'exercice de sa profession ${ }^{[9]}$. Concrètement, le droit au secret professionnel a comme corollaire l'obligation pour le diététiste/nutritionniste de respecter le secret professionnel en tout temps, que ce soit dans l'ensemble de ses communications, dans sa façon de divulguer des informations ou de conserver ses dossiers.

\section{Le respect du secret professionnel et sa portée}

Pour préserver la confidentialité d'un renseignement personnel, il convient d'éviter toute information susceptible d'identifier le client. D'ailleurs, le diététiste/nutritionniste ne doit pas révéler qu'une personne a fait appel à ses services lorsque ce fait est susceptible de causer un préjudice à cette personne, à moins que la loi ne l'ordonne ${ }^{[10]}$. Ce devoir de discrétion s'applique lors des conversations, que ce soit avec son entourage ou avec d'autres clients. Mentionnons à titre d'exemple la radiation d'un ergothérapeute pendant six mois pour divulgation d'informations confidentielles sans autorisation. En effet, il a discuté devant une cliente de l'évolution de ses interventions auprès de certains clients de manière à pouvoir les identifier $^{[11]}$.

Le diététiste/nutritionniste doit éviter les conversations indiscrètes au sujet d'un client et des services qui lui sont rendus ${ }^{[12]}$. Par conséquent, la prudence est de mise lors de discussions relatives à des histoires de cas entre collègues, en personne ou sur les médias sociaux, lors d'activités d'enseignement, de la rédaction d'articles scientifiques ou lors de la diffusion de résultats dans le domaine de la recherche clinique. La même discrétion s'applique dans des situations de marketing. À cet égard, citons le cas d'un médecin qui s'est vu imposer une radiation de deux mois ainsi qu'une amende de $5000 \$$ pour avoir inclus dans un album photo de 
32 bureau ainsi que sur son site Web des photos « avant et après » une intervention chirurgicale sur une patiente sans son consentement ${ }^{[13]}$.

Ainsi, il faut porter une attention particulière aux lieux où sont échangés des renseignements de nature confidentielle et à la manière avec laquelle les services sont discutés. D’ailleurs, le Règlement sur la tenue des cabinets de consultation des diététistes ${ }^{[14]}$ impose d'aménager le cabinet de consultation de façon à ce que l'identité et les conversations des personnes qui s'y trouvent ne puissent être perçues de l'extérieur. Pour illustrer de tels propos, citons le cas d'un médecin condamné à une radiation de deux mois pour avoir tenu une consultation médicale en présence de la fillette de son infirmière sans avoir obtenu préalablement l'approbation et le consentement du patient ${ }^{[15]}$. Ainsi, en raison de l'obligation du respect du secret professionnel, l'aménagement de l'endroit d'exercice du professionnel est soumis à diverses contraintes; les salles de consultation et les salles d'attente doivent offrir un environnement discret qui préserve la nature privée des conversations avec un client. Le devoir d'exercer dans des lieux favorisant la discrétion et le maintien du secret professionnel vise toute forme de «lieux», y compris ceux accessibles par la technologie. Ainsi, lors d'un appel téléphonique ou de l'utilisation d'autres moyens technologiques, il faut proscrire les endroits où on peut être entendu. Il faut s'assurer que les technologies utilisées permettent de préserver le secret professionnel. Dans cette optique, il y a aussi lieu d'être sélectif quant aux informations divulguées lors de l'enregistrement d'un message sur une boite vocale. La même prudence s'impose en utilisant un ordinateur ou en transmettant un courriel : il faut s'assurer que personne n'a accès à l'ordinateur et que seul le destinataire du courriel peut le lire.
Le secret professionnel oblige un professionnel de garder secrètes les informations obtenues de son client dans le cadre de l'exercice de sa profession. Or, les dossiers renferment des renseignements confidentiels de sorte que le diététiste/nutritionniste doit les protéger. Pour assurer la confidentialité des dossiers, le Règlement sur la tenue des cabinets de consultation des diététistes ${ }^{[16]}$ impose de les conserver dans un local ou un meuble auquel le public n'a pas librement accès ; il en est de même s'il utilise l'informatique ou toute autre technique pour la constitution et la tenue de ses dossiers ${ }^{[17]}$. Pour les professionnels exerçant en établissement, la Loi sur les services de santé et les services sociaux ${ }^{[18]}$ stipule expressément que le dossier d'un usager est confidentiel et édicte les règles d'accès devant être respectées ${ }^{[19]}$. Pour ne citer qu'un exemple, mentionnons qu'un ergothérapeute a été radié pendant deux mois pour avoir permis à sa conjointe de prendre connaissance des dossiers de clients afin de l'assister dans la rédaction de ceux-ci et pour ne pas avoir pris les mesures de sécurité propres à assurer la confidentialité des dossiers enregistrés sur une clef USB personnelle ${ }^{[20]}$.

Cet exemple rappelle l'obligation du diététiste/nutritionniste de veiller à ce que ses employés et le personnel qui l'entoure ne révèlent pas les renseignements de nature confidentielle, et ce, en prenant les moyens raisonnables ${ }^{[21]}$. Afin de respecter le secret de tout renseignement de nature confidentielle obtenu dans l'exercice de sa profession, le diététiste/nutritionniste doit régulièrement rappeler à toutes les personnes qui collaborent avec lui qu'elles doivent protéger les informations confidentielles.

Concrètement, tout professionnel doit respecter le secret professionnel à tous égards, en tout temps et en tous lieux. D'ailleurs, en raison de l'importance du secret professionnel, le Code de déontologie des diététistes édicte que le diététiste/nutritionniste ne doit pas faire usage de renseignements de nature confidentielle au préjudice d'un client ou en vue d'obtenir directement ou indirectement un avantage pour lui-même ou pour autrui $^{[22]}$. D'ailleurs, le Conseil de discipline de l'Ordre professionnel des inhalothérapeutes du Québec a reconnu coupable une inhalothérapeute pour avoir consulté à plusieurs reprises, sans motif professionnel, les dossiers médicaux de plus de vingt personnes. Elle a également été trouvée coupable d'avoir accédé à des dossiers médicaux d'au moins deux personnes dans le but de favoriser son intérêt personnel, celle-ci ayant fait des offres d'achat à des patients recevant des soins palliatifs ${ }^{[23]}$. Une consultation ou un usage des dossiers aux seules fins personnelles constitue un bris du secret professionnel; prendre connaissance d'informations confidentielles à des fins personnelles équivaut à les dévoiler à un tiers. L'inhalothérapeute a été radiée pendant six mois et condamnée à une amende de $1000 \$^{[24]}$.

Dans le même ordre d'idées, un travailleur social a été condamné à une radiation d'un mois et à une amende de 1500 \$ pour avoir consulté sans autorisation et sans justification professionnelle des dossiers d'usagers, simplement pour passer le temps ${ }^{[25]}$. Citons aussi le cas d'un physiothérapeute condamné à une amende de 1000 \$ ayant été reconnu coupable d'avoir omis de respecter les renseignements de nature confidentielle portés à sa connaissance. Il avait utilisé le numéro de téléphone cellulaire d'une cliente pour lui envoyer des messages textes à des fins personnelles alors que le numéro avait été obtenu par le système informatique de la clinique où il exerçait ${ }^{[26]}$. Dans un autre cas similaire, un pharmacien a été condamné à une amende de $5000 \$$ après avoir été reconnu coupable d'avoir omis de respecter 
les renseignements de nature confidentielle portés à sa connaissance concernant deux clientes. Il avait utilisé leur numéro de téléphone cellulaire, consigné dans leur dossier pharmacologique, pour les solliciter dans le but de développer une relation intime ${ }^{[27]}$.

Ainsi, le seul statut de professionnel ne donne pas le droit d'accès aux dossiers de clients, ou de patients, lorsqu'aucune raison professionnelle ne le justifie ${ }^{[28]}$.

\section{La levée du secret professionnel}

Bien que chacun ait droit au respect du secret professionnel, ce dernier n'est cependant pas un absolu. Toutes les personnes tenues par la loi au secret professionnel peuvent divulguer les renseignements confidentiels qui leur ont été révélés en raison de leur profession, si elles y sont autorisées par celui qui leur a fait ces confidences ou par une disposition expresse de la loi ${ }^{[29]}$. Ceci est repris par le Code de déontologie des diététistes ${ }^{[30]}$.

Ainsi, il n'existe que deux situations qui permettent de divulguer l'information protégée par le secret professionnel. La première est celle du consentement préalable à la divulgation de la personne qui a fait une confidence. La deuxième est celle où il existe une disposition dans une loi permettant de divulguer certaines informations sans la permission de la personne qui les a communiquées.

Certaines lois autorisent, voire ordonnent la divulgation de l'information confidentielle. En ce qui concerne les diététistes/nutritionnistes, certaines lois permettent la divulgation de renseignements en vue de la protection des personnes. Par conséquent, le diététiste/nutritionniste peut communiquer certains renseignements, même s'il n'en a pas obtenu l'autorisation du patient dans des situations particulières visées par certaines dérogations au secret professionnel prévues dans des lois.
Un professionnel peut communiquer un renseignement protégé par le secret professionnel en vue de prévenir un acte de violence, comme un suicide, lorsqu'il a un motif raisonnable de croire qu'un risque sérieux de mort ou de blessures graves ${ }^{[31]}$ menace une personne donnée ou un groupe de personnes identifiables et que la nature de la menace inspire un sentiment d'urgence ${ }^{[32]}$. Toutefois, le professionnel ne peut alors communiquer ce renseignement qu'aux personnes exposées à ce danger, à leur représentant ou aux personnes susceptibles de leur porter secours. Le professionnel ne peut communiquer que les renseignements nécessaires aux fins poursuivies par la communication. Le Code de déontologie des diététistes reprend cette même possibilité de communication ${ }^{[33]}$. En cas de doute sur le degré d'imminence du danger ou sur ce qu'il convient de faire relativement à la communication, un diététiste/nutritionniste peut consulter un confrère ou une consœur, un membre d'un autre ordre professionnel ou toute autre personne compétente, à condition que cette consultation n'entraîne pas de retard préjudiciable à la communication ${ }^{[34]}$. Pour juger du risque de la situation, il peut être pertinent de consulter par exemple un travailleur social, un psychologue ou un psychiatre.

Également, tout professionnel qui s'occupe d'un enfant a une obligation de signalement sans délai à un directeur de la protection de la jeunesse s'il a un motif raisonnable de croire que la sécurité ou le développement d'un enfant est ou peut être considéré comme compromis ${ }^{[35]}$. De même pour les personnes hébergées dans un Centre d'hébergement et de soins de longue durée et les personnes inaptes ${ }^{[36]}$, il existe le signalement obligatoire et sans délai par tout professionnel qui a un motif raisonnable de croire qu'une telle personne est victime d'un geste singulier ou répétitif ou de l'absence d'intervention appropriée portant atteinte de façon sérieuse à son intégrité physique ou psychologique ${ }^{[37]}$. Pour plus de détails sur ces signalements, nous invitons le lecteur à consulter une autre chronique juridique parue dans la revue de l'OPDQ ${ }^{[38]}$.

Donc, malgré l'importance du secret professionnel, un professionnel doit parfois concilier le respect de ce secret professionnel avec d'autres préoccupations tout aussi importantes, notamment la sécurité des tiers. Toutefois, ce ne sont pas toutes les préoccupations qui permettent d'outrepasser le secret professionnel. Une infirmière l'a appris à ses dépens après avoir filmé des patients et avoir transmis la vidéo à un média pour dénoncer une situation sans avoir obtenu leur autorisation. On lui a ainsi reproché de ne pas avoir respecté le secret professionnel. Elle s'est vu imposer une radiation temporaire de deux mois ${ }^{[39]}$.

Enfin, parmi les exceptions au respect du secret professionnel, il y a celles prévoyant que le diététiste/ nutritionniste ne peut se retrancher derrière le paravent de ce secret lorsqu'il doit divulguer des informations, notamment au comité de l'inspection professionnelle ${ }^{[40]}$, au syndic ${ }^{[41]}$ ou encore au Conseil de discipline ${ }^{[42]}$ de l'OPDQ.

\section{L'échange d'informations confidentielles}

À l'instar de nombreux domaines, dans le domaine de la santé, l'exercice d'une profession étant de plus en plus complexe et spécialisé, il est fréquent qu'un professionnel recherche de l'aide auprès d'autres professionnels, voire qu'il veuille échanger sur diverses informations. De surcroît, toute équipe médicale ne peut efficacement rendre ses services que dans la mesure où chaque professionnel prend connaissance de l'ensemble des renseignements nécessaires. Il faut alors un certain partage du secret, c'est-à-dire des informations 
34 obtenues sous le sceau de la confidentialité. D'ailleurs, le concept de secret partagé s'applique lors de démarches pour assurer la continuité des soins lors d'un transfert ou d'une consultation, ces événements impliquant la levée du secret professionnel ${ }^{[43]}$. Ce n'est donc pas une faute de la part du professionnel que de faire part à d'autres professionnels des renseignements confidentiellement obtenus pour assurer la poursuite des soins. La communication d'informations confidentielles entre divers intervenants appelés à collaborer dans la prestation des soins ou des services au bénéfice d'un client ne constitue pas une violation du droit à la confidentialité dans la mesure où il s'agit de la communication d'informations nécessaires et pertinentes à l'exécution des fonctions de chacun des membres d'une équipe de soins et de services.

Ainsi, ce n'est pas parce qu'un patient dévoile des informations qu'il veut garder confidentielles qu'il ne faut pas communiquer ce genre de renseignements, dans la mesure où il s'agit d'informations pertinentes pour les interventions des autres professionnels. D’ailleurs, un infirmier a été reconnu coupable d'avoir omis d'informer le reste de l'équipe soignante d'une unité de psychiatrie qu'il avait reçu des appels téléphoniques d'une patiente qui lui avait fait part de son inquiétude et de son désarroi, alors qu'il avait été statué que l'infirmier devait communiquer à l'équipe tout renseignement ou confidence d'un patient; on lui a imposé une radiation de deux semaines ${ }^{[44]}$.

Cela dit, la communication d'informations n'est pas sans limites ; malgré toutes les informations pouvant se trouver dans un dossier, rappelons que chaque intervenant doit s'astreindre à une discipline personnelle pour limiter son accès aux seuls extraits pertinents et nécessaires à ses tâches ${ }^{[45]}$. Néanmoins, on reconnaît au personnel d'un établissement le droit d'obtenir l'accès à un dossier lorsque cela est nécessaire et pertinent dans l'exercice des fonctions ${ }^{[46]}$.

\section{Conclusion}

Le devoir de respecter le secret professionnel existe, quel que soit le milieu de pratique. Le secret professionnel concerne donc tous les diététistes/nutritionnistes, peu importe le secteur d'activités. Ainsi, peu importe son domaine d'exercice, un diététiste/nutritionniste doit avoir le souci constant de la confidentialité des informations obtenues, que ce soit les déclarations et les faits qui lui sont rapportés ou les constatations qu'il fait lui-même.

N.D.L.R.* L'auteure est diététiste/ nutritionniste, avocate et présidente du comité sur la pratique illégale de l'Ordre professionnel des diététistes du Québec.

\section{Références}

1. En 2003, le secret professionnel avait fait l'objet d'un article dans le premier numéro de la présente revue Nutrition, science et évolution dont l'ancêtre était Diététique en Action. Voir: Janick Perreault, «Le secret professionnel», Nutrition, science et évolution, vol. 1, no 1, printemps 2003.

2. RLRQ, c. A-2.1.

3. RLRQ, c. S-4.2.

4. RLRQ, P-39.1.

5. 5 RLRQ, c. C-12, art. 9.

6. Code des professions, RLRQ, c. C-26.

7. Id., art. 60.4.

8. Code de déontologie des diététistes, RLRQ, c. C-26, r. 65.01.

9. Id., art. 24.

10. Id., art. 26

11. Ergothérapeutes (Ordre professionnel des) c. Dumas, 17-04-00001, 25 janvier 2005 (QC OEQ)

12. Code de déontologie des diététistes, art. 27.

13. Médecins (Ordre professionnel des) c. Benchetrit, 24-13-00811, 29 juillet 2015 (QC CDCM).

14. Règlement sur la tenue des cabinets de consultation des diététistes c. C-26, r. 74, art. 2.01.

15. Médecins (Ordre professionnel des) c. Courchesne, 24-14-00857, 30 juin 2016 (QC CDCM).

16. Règlement sur la tenue des dossiers des diététistes, chapitre C-26, r. 105.

17. Id., art. 2.05 .

18. Loi sur les services de santé et les services sociaux, RLRQ, C. s. 4.2

19. Id., art. 19

20. Ergothérapeutes (Ordre professionnel des) c. Jean, 17-13-00028, 28 avril 2014 (QC OEQ).
21. Code de déontologie des diététistes, art. 29.

22. Code de déontologie des diététistes, art. 28.

23. Elle voulait acheter à bas prix la maison d'un patient en fin de vie, voir: http://www. lapresse.ca/actualites/justice-et-faits-divers/201801/28/01-5151761-elle-voulait-achetera-bas-prix-la-maison-dun-patient-en-fin-de-vie. php.

24. Inhalothérapeutes (Ordre professionnel des) c. Milmore, 40-16-038, 29 mars 2017 (QC OPIQ); décision sur sanction le 11 janvier 2018.

25. Travailleurs sociaux (Ordre professionnel des) c. Rochette, 37-11-006, 29 juin 2012 (QC OTSTCFQ).

26. Physiothérapie (Ordre professionnel de la) c. Landreville, 31-15-009, 20 avril 2016 (QC OPPQ).

27. Pharmaciens (Ordre professionnel des) c. Bédard, 30-17-01895, 25 juillet 2018 (QC CDOPQ).

28. Inhalothérapeutes (Ordre professionnel des) c. Milmore, 40-16-038, 29 mars 2017 par. 32 (QC OPIQ); décision sur sanction le 11 janvier 2018.

29. Code des professions, RLRQ, c. C-26, art. 60.4, al. 2.

30. Code de déontologie des diététistes, RLRQ, c. C-26, r. 65.01, art. 25. Également, Charte des droits et libertés de la personne, art. 9; Code des professions, art. 60.4.

31. On entend par «blessures graves» toute blessure physique ou psychologique qui nuit d'une manière importante à l’intégrité physique, à la santé ou au bien-être d'une personne ou d'un groupe de personnes identifiable.

32. Code des professions, RLRQ, c. C-26, art. 60.4, al. 3.

33. Code de déontologie des diététistes, art. 29.1. 34. Id., art. 29.2

35. Loi sur la protection de la jeunesse, RLRQ, chapitre P-34.1, art. 39.

36. Il s'agit des personnes en tutelle ou en curatelle ou à l'égard desquelles un mandat de protection a été homologué.

37. Loi visant à lutter contre la maltraitance envers les aînés et toute autre personne majeure en situation de vulnérabilité, RLRQ, chapitre L-6.3, art. 21.

38. Janick Perreault, «Bientraitance des adultes: Une nouvelle loi pour lutter contre la maltraitance envers les aînés et les autres personnes majeures en situation de vulnérabilité», Chronique juridique de la Revue de l'Ordre professionnel des diététistes du Québec, Nutrition, science et évolution, vol. 15, no 2, hiver 2018

39. Infirmières et infirmiers (Ordre professionnel des) c. Tremblay, 20-2007-00391, 14 novembre 2008 (QC CDOII).

40. Code des professions, art. 114, et 192

41. Id., art. 122, et 192

42. Id., 147, 149, 174 et 192.

43. Suzanne Philips-Nootens, Robert P. Khouri et Pauline Lesage-Jarjoura, Éléments de responsabilité civile médicale, 4ième éd., Cowansville, Éditions Yvon Blais, 2016, ${ }^{\circ}$ 415, page 388.

44. Desmeules c. Infirmiers et infirmières, 2002 QCTP 071, 18 juin 2002.

45. Inhalothérapeutes (Ordre professionnel des) c. Milmore, 40-16-038, 29 mars 2017, par. 35 (QC OPIQ); décision sur sanction le 11 janvier 2018.

46. Id., par. 34 